Proyecciones Journal of Mathematics

Vol. 34, No 1, pp. 25-44, March 2015.

Universidad Católica del Norte

Antofagasta - Chile

\title{
Stability in totally nonlinear neutral differential equations with variable delay using fixed point theory
}

\author{
Abdelouaheb Ardjouni \\ University Souk Ahras, Algeria \\ and \\ Ahcene Djoudi \\ University Annaba, Algeria \\ Received: September 2014. Accepted : December 2014
}

\begin{abstract}
The totally nonlinear neutral differential equation

$$
\frac{d}{d t} x(t)=-a(t) g(x(t-\tau(t)))+\frac{d}{d t} G(t, x(t-\tau(t))),
$$

with variable delay $\tau(t) \geq 0$ is investigated. We find suitable conditions for $\tau, a, g$ and $G$ so that for a given continuous initial function $\psi$ a mapping $P$ for the above equation can be defined on a carefully chosen complete metric space $S_{\psi}^{0}$ and in which $P$ possesses a unique fixed point. The final result is an asymptotic stability theorem for the zero solution with a necessary and sufficient condition. The obtained theorem improves and generalizes previous results due to Becker and Burton [6]. An example is given to illustrate our main result.
\end{abstract}

Subjclass[2010] : 34K20, 34K30, 34K40.

Keywords : Fixed points, Stability, Neutral differential equations, Variable delays. 


\section{Introduction}

Without doubt, Lyapunov's direct method has been, for more than 100 years, the most efficient tool for investigating the stability properties of a wide variety of ordinary, functional, partial differential and integro-differential equations. Nevertheless, the application of this method to problems of stability in differential and integro-differential equations with delays has encountered serious obstacles if the delays are unbounded or if the equation has unbounded terms [8]-[10]. In recent years, several investigators have tried stability by using a new technique. Particularly, Burton, Furumochi, Becker, Zhang and others began a study in which they noticed that some of these difficulties vanish or might be overcome by means of fixed point theory (see [1]-[21], [23]-[25]). The fixed point theory does not only solve the problem on stability but has other significant advantage over Lyapunov's. The conditions of the former are often averages but those of the latter are usually pointwise (see [8]). Moreover, the fixed point method have been successfully used to conclude stability results to delay problems which are perturbed by stochastic terms (see for example [20]). This is another important feature for applications to real-world problems.

In this paper we focus on the totally nonlinear neutral differential equation with variable delay

$$
\frac{d}{d t} x(t)=-a(t) g(x(t-\tau(t)))+\frac{d}{d t} G(t, x(t-\tau(t))),
$$

with the initial condition

$$
x(t)=\psi(t) \text { for } t \in[m(0), 0],
$$

such that $\psi \in C([m(0), 0]$,$) where m(0)=\inf \{t-\tau(t), t \geq 0\}$.

Here $C\left(S_{1}, S_{2}\right)$ denotes the set of all continuous functions $\varphi: S_{1} \rightarrow S_{2}$ with the supremum norm $\|\cdot\|$. Throughout this paper we assume that $a \in C\left(\mathbf{R}^{+}, \mathbf{R}\right)$ and $\tau: \mathbf{R}^{+} \rightarrow \mathbf{R}^{+}$is differentiable with $t-\tau(t) \rightarrow \infty$ as $t \rightarrow \infty$. The function $G(t, x)$ is globally Lipschitz continuous in $x$. That is, there is positive constant $E$ such that

$$
|G(t, x)-G(t, y)| \leq E|x-y|
$$

We also assume that

$$
G(t, 0)=0
$$


Special cases of (1.1) have been considered and investigated by many authors. For example the equation

$$
x^{\prime}(t)=-a(t) g(x(t-\tau(t))),
$$

is of historical importance and has significant applications. The study of (1.4) go back to 1951 (cf. [23] and the references therein) and it has attracted the attention of a large number of investigators. To our knowledge, the most recent work about this equation has been done by Becker and Burton in [6]. In this paper the authors have established the following result.

Theorem 1 (Becker and Burton [6]). Suppose that there exists a constant $l>0$ such that $g$ satisfies a Lipschitz condition on $[-l, l]$. Assume, further, that

(i) the function $t-\tau(t):[0, \infty) \rightarrow[m(0), \infty)$ is strictly increasing;

(ii) $g$ is odd and strictly increasing on $[-l, l]$;

(iii) $x-g(x)$ is non-decreasing on $[0, l]$;

(iv) there is an $\alpha \in(0,1)$ such that

$$
2 \int_{t-\tau(t)}^{t} a(u) d u \leq \alpha \text { for } t \geq t_{1}
$$

where $t_{1}$ is the unique solution of $t-\tau(t)=0$, and a continuous function $\widetilde{a}:[0, \infty) \rightarrow \mathbf{R}$ exists such that $a(t)=\widetilde{a}(t)\left(1-\tau^{\prime}(t)\right)$ on $[0, \infty)$.

Then a $\delta \in(0, l)$ exists such that, for each continuous function $\psi:[m(0), 0] \rightarrow$ $(-\delta, \delta)$, there is a unique continuous function $x:[m(0), \infty) \rightarrow \mathbf{R}$ with $x(t)=\psi(t)$ on $[m(0), 0]$ that satisfies (1.4) on $[0, \infty)$. Moreover, $x$ is bounded by $l$ on $[m(0), \infty)$. Furthermore, the zero solution of (1.4) is stable at $t=0$. If, in addition, $g$ is continuously differentiable, $g^{\prime}(0) \neq 0$ and

$$
\int_{0}^{t} a(v) d v \rightarrow \infty \text { as } t \rightarrow \infty
$$

then the zero solution is asymptotically stable.

Our purpose here is to improve Theorem 1 and extend it to support a wide class of nonlinear differential equation with variable delay of neutral type presented in (1.1). We point out here that the present result does not require that $t-\tau(t)$ be strictly increasing. Such assumption has been at the heart of the methods used in the previous papers and here we propose a way with no need of further hypotheses on the inverse of delay $t-\tau(t)$, 
so that for a given continuous initial function $\psi$ a mapping $P$ for (1.1) is constructed in such a way to map a, carefully chosen, complete metric space $S_{\psi}^{0}$ into itself and on which $P$ is a contraction mapping possessing a fixed point. This procedure will enable us to establish and prove by means of the contraction mapping theorem an asymptotic stability theorem for the zero solution of (1.1) with less restrictive conditions. The main results improve and generalize the main results in [6]. We also provide an example to illustrate our claim.

\section{Main results}

For each $\psi \in C([m(0), 0], \mathbf{R})$, a solution of $(1.1)$ through $(0, \psi)$ is a continuous function $x:[m(0), T) \rightarrow \mathbf{R}$ for some positive constant $T>0$ such that $x$ satisfies $(1.1)$ on $[0, T)$ and $x=\psi$ on $[m(0), 0]$. We denote such a solution by $x(t)=x(t, 0, \psi)$. From the existence theory we know that for each $\psi \in C([m(0), 0], \mathbf{R})$, there exists a unique solution $x(t)=x(t, 0, \psi)$ of $(1.1)$ defined on $[0, \infty)$. We define $\|\psi\|:=\max \{|\psi(t)|: m(0) \leq t \leq 0\}$.

The absence of linear terms in (1.1) makes it difficult to obtain a fixed point mapping for (1.1). So, to make (1.1) more tractable, we have to transform it.

Generally, the investigation of the stability of an equation using fixed point technique involves the construction of a suitable fixed point mapping. This can, in so many cases, be a difficult task. So, we begin by transforming (1.1) to a more tractable, but equivalent, equation which we then invert to obtain an equivalent integral equation from which we derive a fixed point mapping. After then, we define a suitable complete space depending on the initial condition, so that the mapping is a contraction. Using Banach's contraction mapping principle [22], we obtain a solution for this mapping and hence a solution for (1.1), which is asymptotically stable.

First, we begin by transforming (1.1) into an equivalent equation to which we apply the variation of parameters to define a fixed point mapping.

Lemma 1. Let $\psi:[m(0), 0] \rightarrow \mathbf{R}$ be a given continuous initial function. If $x$ is a solution of (1.1) on an interval $[0, T)$ with $x=\psi$ on $[m(0), 0]$, then $x$ is a solution of the integral equation

$$
\begin{aligned}
& x(t)=\left\{\psi(0)-G(0, \psi(-\tau(0)))-\int_{-\tau(0)}^{0} H(s) g(\psi(s)) d s\right\} e^{-\int_{0}^{t} H(v) d v} \\
& +G(t, x(t-\tau(t)))+\int_{t-\tau(t)}^{t} H(s) g(x(s)) d s
\end{aligned}
$$




$$
\begin{aligned}
& -\int_{0}^{t} e^{-\int_{s}^{t} H(v) d v} H(s)\left(\int_{s-\tau(s)}^{s} H(u) g(x(u)) d u\right) d s \\
& +\int_{0}^{t} e^{-\int_{s}^{t} H(v) d v}\left\{\left[-a(s)+H(s-\tau(s))\left(1-\tau^{\prime}(s)\right)\right] g(x(s-\tau(s)))\right. \\
& -H(s) G(s, x(s-\tau(s)))\} d s \\
& \quad+\int_{0}^{t} e^{-\int_{s}^{t} H(v) d v} H(s)[x(s)-g(x(s))] d s,
\end{aligned}
$$

where $H:[m(0), \infty) \rightarrow \mathbf{R}$ is an arbitrary continuous function. Conversely, if a continuous function $x$ is equal to $\psi$ on $[m(0), 0]$ and is a solution of (2.1) on an interval $[0, \sigma)$, then $x$ is a solution of $(1.1)$ on $[0, \sigma)$.

Proof. Let $x$ be a solution of (1.1). Rewrite (1.1) in the following equivalent form

$$
\begin{aligned}
& \frac{d}{d t}\{x(t)-G(t, x(t-\tau(t)))\} \\
& =-H(t)[x(t)-G(t, x(t-\tau(t)))]+\frac{d}{d t} \int_{t-\tau(t)}^{t} H(s) g(x(s)) d s \\
& -a(t) g(x(t-\tau(t)))+H(t-\tau(t))\left(1-\tau^{\prime}(t)\right) g(x(t-\tau(t))) \\
& \quad-H(t) G(t, x(t-\tau(t)))+H(t)[x(t)-g(x(t))] .
\end{aligned}
$$

Multiplying both sides of (2.2) by the factor $e^{\int_{0}^{t} H(v) d v}$ and integrating from 0 to any $t \in[0, T)$, we obtain

$$
\begin{aligned}
& \mathrm{x}(\mathrm{t})=(\psi(0)-G(0, \psi(-\tau(0)))) e^{-\int_{0}^{t} H(v) d v}+G(t, x(t-\tau(t))) \\
& +\int_{0}^{t} e^{-\int_{s}^{t} H(v) d v} \frac{d}{d s}\left(\int_{s-\tau(s)}^{s} H(s) g(x(u)) d u\right) d s \\
& +\int_{0}^{t} e^{-\int_{s}^{t} H(v) d v}\left[-a(s)+H(s-\tau(s))\left(1-\tau^{\prime}(s)\right)\right] g(x(s-\tau(s))) d s \\
& -\int_{0}^{t} e^{-\int_{s}^{t} H(v) d v} H(s) G(s, x(s-\tau(s))) d s \\
& +\int_{0}^{t} e^{-\int_{s}^{t} H(v) d v} H(s)[x(s)-g(x(s))] d s .
\end{aligned}
$$

Using integration by parts, we conclude that

$$
x(t)=\left\{\psi(0)-G(0, \psi(-\tau(0)))-\int_{-\tau(0)}^{0} H(s) g(\psi(s)) d s\right\} e^{-\int_{0}^{t} H(v) d v}
$$




$$
\begin{aligned}
& +G(t, x(t-\tau(t)))+\int_{t-\tau(t)}^{t} H(s) g(x(s)) d s \\
- & \int_{0}^{t} e^{-\int_{s}^{t} H(v) d v} H(s)\left(\int_{s-\tau(s)}^{s} H(u) g(x(u)) d u\right) d s \\
& +\int_{0}^{t} e^{-\int_{s}^{t} H(v) d v}\left\{\left[-a(s)+H(s-\tau(s))\left(1-\tau^{\prime}(s)\right)\right] g(x(s-\tau(s)))\right. \\
& -H(s) G(s, x(s-\tau(s)))\} d s \\
& +\int_{0}^{t} e^{-\int_{s}^{t} H(v) d v} H(s)[x(s)-g(x(s))] d s .
\end{aligned}
$$

Conversely, suppose that a continuous function $x$ is equal to $\psi$ on $[m(0), 0]$ and satisfies (2.1) on an interval $[0, \sigma)$. Then, $x$ is differentiable on $[0, \sigma)$. Differentiating $x$ with the aid of Leibniz's rule, we obtain (1.1).

From equation (2.1) we shall derive a fixed point mapping $P$ for (1.1). But the challenge here is to choose a suitable metric space of functions on which the map $P$ can be defined. Below a weighted metric on a specific space is defined. Let $C$ be the set of real-valued bounded continuous functions on $[m(0), \infty)$ with the supremum norm $\|\cdot\|$, that is, for $\phi \in C$,

$$
\|\phi\|:=\sup \{|\phi(t)|: t \in[m(0), \infty)\} .
$$

In other words, we carry out our investigations in the complete metric space $(C, d)$ where $d$ denotes the supremum metric $d\left(\phi_{1}, \phi_{2}\right)=\left\|\phi_{1}-\phi_{2}\right\|$ for $\phi_{1}, \phi_{2} \in C$. For a given initial function $\psi:[m(0), 0] \rightarrow[-l, l], l>0$, define the set

$S_{\psi}:=\{\phi:[m(0), \infty) \rightarrow \mathbf{R} \mid \phi \in C, \phi(t)=\psi(t)$ for $t \in[m(0), 0],|\phi(t)| \leq l\}$.

Since $S_{\psi}$ is a closed subset of $C$, the metric space $\left(S_{\psi}, d\right)$ is complete.

Theorem 2. Let $H:[m(0), \infty) \rightarrow \mathbf{R}$ be a continuous function and define a mapping $P$ on $S_{\psi}$ as follows, for $\phi \in S_{\psi}$,

$$
(P \phi)(t)=\psi(t) \text { if } t \in[m(0), 0],
$$

while for $t>0$,

$$
\begin{aligned}
& (P \phi)(t)=\left\{\psi(0)-G(0, \psi(-\tau(0)))-\int_{-\tau(0)}^{0} H(s) g(\psi(s)) d s\right\} e^{-\int_{0}^{t} H(v) d v} \\
& +G(t, \phi(t-\tau(t)))+\int_{t-\tau(t)}^{t} H(s) g(\phi(s)) d s
\end{aligned}
$$




$$
\begin{aligned}
& -\int_{0}^{t} e^{-\int_{s}^{t} H(v) d v} H(s)\left(\int_{s-\tau(s)}^{s} H(u) g(\phi(u)) d u\right) d s \\
& +\int_{0}^{t} e^{-\int_{s}^{t} H(v) d v}\left\{\left[-a(s)+H(s-\tau(s))\left(1-\tau^{\prime}(s)\right)\right] g(\phi(s-\tau(s)))\right. \\
& -H(s) G(s, \phi(s-\tau(s)))\} d s \\
& \quad+\int_{0}^{t} e^{-\int_{s}^{t} H(v) d v} H(s)[\phi(s)-g(\phi(s))] d s .
\end{aligned}
$$

Suppose that (1.2) holds and the following conditions are satisfied, (i) there exists a constant $l>0$ such that $g$ satisfies a Lipschitz condition on $[-l, l]$ and let $L$ be the Lipschitz constant for both $g(x)$ and $x-g(x)$ on $[-l, l]$

(ii) $H(t) \geq 0$ for $t \geq m(0)$.

Assume, further, that the following condition is satisfied for some constant $k>5$

$$
k E \leq 1,
$$

where $E$ is given by (1.2). Then there is a metric $d_{h}$ for $S_{\psi}$ such that $\left(S_{\psi}, d_{h}\right)$ is complete and $P$ is a contraction on $\left(S_{\psi}, d_{h}\right)$ if $P$ maps $S_{\psi}$ into itself.

Proof. It is clear that $P \phi$ is continuous. Now, for $t \in[m(0), \infty)$ and a constant $k>5$, define

$$
h(t):=k L \int_{0}^{t}[H(v)+\omega(v)] d v
$$

where

$$
\omega(v):=\left\{\begin{array}{l}
0, \quad \text { if } v \in[m(0), 0] \\
\left|-a(v)+H(v-\tau(v))\left(1-\tau^{\prime}(v)\right)\right|+\frac{E H(v)}{L}, \text { if } v \in[0, \infty) .
\end{array}\right.
$$

Let $S$ be the space of all continuous functions $\phi:[m(0), \infty) \rightarrow \mathbf{R}$ such that

$$
|\phi|_{h}:=\sup \left\{|\phi(t)| e^{-h(t)}: t \in[m(0), \infty)\right\}<\infty .
$$


Then $\left(S,|\cdot|_{h}\right)$ is a Banach space, which can be checked with Cauchy criterion for uniform convergence. Thus, $\left(S, d_{h}\right)$ is a complete metric space where $d_{h}$ denotes the induced metric $d_{h}(\phi, \eta)=|\phi-\eta|_{h}$ for $\phi, \eta \in S$. Being closed in $S$ with this metric, the space $\left(S_{\psi},|\cdot|_{h}\right)$ is also complete. Suppose now, that $P: S_{\psi} \rightarrow S_{\psi}$. We need to show that $P$ defined by (2.3) is a contraction. Toward this end, let $\phi, \eta \in S_{\psi}$, since, by (1.2) and (i), $g$ and $G$ satisfy Lipschitz conditions on $[-l, l]$, it follows that

$$
\begin{aligned}
& \quad|(P \phi)(t)-(P \eta)(t)| e^{-h(t)} \\
& \leq E|\phi(t-\tau(t))-\eta(t-\tau(t))| e^{-h(t)-h(t-\tau(t))+h(t-\tau(t))} \\
& +\int_{t-\tau(t)}^{t} H(s) L|\phi(s)-\eta(s)| e^{-h(t)+h(s)-h(s)} d s \\
& +\int_{o}^{t} e^{-\int_{s}^{t} H(v) d v} H(s) \int_{s-\tau(s)}^{s} H(u) L|\phi(u)-\eta(u)| e^{-h(t)+h(u)-h(u)} d u d s \\
& +\int_{0}^{t} e^{-\int_{s}^{t} H(v) d v}\left\{L\left|-a(s)+H(s-\tau(s))\left(1-\tau^{\prime}(s)\right)\right|+E H(s)\right\} \\
& \times|\phi(s-\tau(s))-\eta(s-\tau(s))| e^{-h(t)+h(s-\tau(s))-h(s-\tau(s))} d s \\
& \quad+\int_{0}^{t} e^{-\int_{s}^{t} H(v) d v} H(s) L|\phi(s)-\eta(s)| e^{-h(t)+h(s)-h(s)} d s,
\end{aligned}
$$

for $t>0$. There are five terms on the right hand side of inequality (2.6), denote them respectively by $I_{i}, i=1,2, \ldots, 5$. For $t-\tau(t) \leq s \leq v \leq t$, we have

$$
-h(t)+h(s)=-k L \int_{s}^{t}[H(v)+\omega(v)] d v \leq-k L \int_{s}^{t} H(v) d v .
$$

Consequently,

$$
I_{2} \leq \int_{t-\tau(t)}^{t} e^{-k L \int_{s}^{t} H(v) d v} H(s) L|\phi(s)-\eta(s)| e^{-h(s)} d s .
$$

For $s-\tau(s) \leq u \leq v \leq s$, we have

$$
-h(t)+h(u)=-k L \int_{u}^{t}[H(v)+\omega(v)] d v \leq-k L \int_{u}^{s} H(v) d v .
$$


So

$I_{3} \leq \int_{0}^{t} e^{-\int_{s}^{t} H(v) d v} H(s) \int_{s-\tau(s)}^{s} e^{-k L \int_{u}^{s} H(v) d v} H(u) L|\phi(u)-\eta(u)| e^{-h(u)} d u d s$.

Similarly, using (2.5), we obtain for $s-\tau(s) \leq t$

$$
-h(t)+h(s-\tau(s))=-k L \int_{s-\tau(s)}^{t}[H(v)+\omega(v)] d v \leq-k L \int_{s}^{t} \omega(v) d v .
$$

Thus

$$
I_{4} \leq \int_{0}^{t} e^{-k L \int_{s}^{t} \omega(v) d v} \omega(s) L|\phi(s-\tau(s))-\eta(s-\tau(s))| e^{-h(s-\tau(s))} d s .
$$

Consequently, inequality $(2.6)$, became $|(P \phi)(t)-(P \eta)(t)| e^{-h(t)}$

$$
\begin{aligned}
& \leq e^{-k L \int_{t-\tau(t)}^{t}[H(v)+\omega(v)] d v} E|\phi(t-\tau(t))-\eta(t-\tau(t))| e^{-h(t-\tau(t))} \\
& +\int_{t-\tau(t)}^{t} e^{-k L \int_{u}^{t} H(v) d v} H(s) L|\phi(s)-\eta(s)| e^{-h(s)} d s \\
& +\int_{0}^{t} e^{-\int_{s}^{t} H(v) d v} H(s) \int_{s-\tau(s)}^{s} e^{-k L \int_{u}^{s} H(v) d v} H(u) L|\phi(u)-\eta(u)| e^{-h(u)} d u d s \\
& +\int_{0}^{t} e^{-k L \int_{s}^{t} \omega(v) d v} \omega(s) L|\phi(s-\tau(s))-\eta(s-\tau(s))| e^{-h(s-\tau(s))} d s \\
& +\int_{0}^{t} e^{-(k L+1) \int_{s}^{t} H(v) d v} H(s) L|\phi(s)-\eta(s)| e^{-h(s)} d s .
\end{aligned}
$$

Consequently, by using (2.4), we obtain

$$
|(P \phi)(t)-(P \eta)(t)| e^{-h(t)} \leq\left[\frac{1}{k}+\left(\frac{1}{k L}+\frac{1}{k L}+\frac{1}{k L}+\frac{1}{k L+1}\right) L\right]|\phi-\eta|_{h}
$$

for all $t>0$. Since $P \phi$ and $P \eta$ agree on $[m(0), 0]$, the last inequality holds for all $t \geq m(0)$.

Thus

$$
|P \phi-P \eta|_{h} \leq \frac{5}{k}|\phi-\eta|_{h} .
$$

Since $k>5$, we conclude that $P$ is a contraction on $\left(S_{\psi}, d_{h}\right)$.

Below, we choose $\|\psi\|$ sufficiently small to establish the existence and uniqueness of solutions by showing that $P: S_{\psi} \rightarrow S_{\psi}$. Furthermore, we prove that the zero solution of (1.1) satisfies the following definition. 
Definition 1. The zero solution of (1.1) is said to be stable at $t=0$ if, for every $\varepsilon>0$, there exists a $\delta>0$ such that $\psi:[m(0), 0] \rightarrow(-\delta, \delta)$ implies that $|x(t)|<\varepsilon$ for $t \geq m(0)$.

Theorem 3. Suppose $g$ and $H$ satisfy conditions (i)-(iii) in Theorem 2, (1.2), (1.3) and (2.4) hold, and suppose further that

(i) $g$ is odd and strictly increasing on $[-l, l]$;

(ii) $x-g(x)$ is non-decreasing on $[0, l]$;

(iii) there exists an $\alpha \in(0,1)$ such that, for $t \geq 0$

$$
\begin{aligned}
& 2 l E+g(l)\left(\int_{t-\tau(t)}^{t} H(s) d s+\int_{0}^{t} e^{-\int_{s}^{t} H(v) d v} H(s)\left(\int_{s-\tau(s)}^{s} H(u) d u\right) d s\right. \\
& \left.+\int_{0}^{t} e^{-\int_{s}^{t} H(v) d v}\left|-a(s)+H(s-\tau(s))\left(1-\tau^{\prime}(s)\right)\right| d s\right) \\
& \leq \alpha g(l) .
\end{aligned}
$$

Then a $\delta \in(0, l)$ exists such that for each initial continuous function $\psi$ : $[m(0), 0] \rightarrow(-\delta, \delta)$, there is a unique continuous function $x:[m(0), \infty) \rightarrow$ $\mathbf{R}$ with $x=\psi$ on $[m(0), 0]$, which is a solution of $(1.1)$ on $[0, \infty)$. Moreover, $x$ is bounded by $l$ on $[m(0), \infty)$. Furthermore, the zero solution of $(1.1)$ is stable at $t=0$.

Proof. Since $g$ is odd and satisfies the Lipschitz condition on $[-l, l]$, $g(0)=0$ and $g$ is uniformly continuous on $[-l, l]$. So we can choose a $\delta$ that satisfies

$$
\delta(1+E)+g(\delta) \int_{-\tau(0)}^{0} H(s) d s \leq(1-\alpha) g(l) .
$$

Let $\psi:[m(0), 0] \rightarrow(-\delta, \delta)$ be a continuous function. Note that (2.7) implies $\delta<l$ since $g(l) \leq l$ by condition (ii). Thus, $|\psi(t)| \leq l$ for $m(0) \leq t \leq 0$. Now we show that for such a $\psi$ the mapping $P: S_{\psi} \rightarrow S_{\psi}$. Indeed, consider (2.3). For an arbitrary $\phi \in S_{\psi}$, it follows from (1.2), (1.3) and conditions (i) and (ii) that

$$
\begin{aligned}
& |(P \phi)(t)| \\
& \leq \delta(1+E)+g(\delta) \int_{-\tau(0)}^{0} H(s) d s+l E \\
& +g(l) \int_{t-\tau(t)}^{t} H(s) d s+g(l) \int_{o}^{t} e^{-\int_{s}^{t} H(v) d v} H(s)\left(\int_{s-\tau(s)}^{s} H(u) d u\right) d s
\end{aligned}
$$




$$
\begin{aligned}
& +\int_{0}^{t} e^{-\int_{s}^{t} H(v) d v}\left\{g(l)\left|-a(s)+H(s-\tau(s))\left(1-\tau^{\prime}(s)\right)\right|+l E H(s)\right\} d s \\
& +(l-g(l)) \int_{0}^{t} e^{-\int_{s}^{t} H(v) d v} H(s) d s
\end{aligned}
$$

for $t>0$. By applying (iii) and (2.7), we see that

$$
\begin{aligned}
& |(P \phi)(t)| \leq \delta(1+E)+g(\delta) \int_{-\tau(0)}^{0} H(s) d s+\alpha g(l)+l-g(l) \\
& \leq(1-\alpha) g(l)+\alpha g(l)+l-g(l)=(1-\alpha) g(l)+(\alpha-1) g(l)+l=l .
\end{aligned}
$$

Hence, $|(P \phi)(t)| \leq l$ for $t \in[m(0), \infty)$ because $|(P \phi)(t)|=|\psi(t)| \leq l$ for $t \in[m(0), 0]$. Therefore, $P \phi \in S_{\psi}$. By Theorem 2, $P$ is a contraction on the complete metric space $\left(S_{\psi}, d_{h}\right)$. Then $P$ has a unique fixed point $x \in S_{\psi}$. Thus $|x(t)| \leq l$ for all $t \geq m(0)$ and is a solution of $(1.1)$ on $[0, \infty)$ by Lemma 1 . Hence $x$ is the only continuous function satisfying (1.1) such that $x(t)=\psi(t)$ for $t \in[m(0), 0]$.

To prove the stability at $t=0$, let $\varepsilon>0$ be given and choose $r>0$ such that $r<\min \{\varepsilon, l\}$. Replacing $l$ with $r$ beginning with (2.7), we see that there is a $\delta>0$ such that $\|\psi\|<\delta$ implies that the unique continuous solution $x$ agreeing on $[m(0), 0]$ with $\psi$, satisfies $|x(t)| \leq r<\varepsilon$ for all $t m(0)$.

Supposing that the conditions in Theorem 2 and Theorem 3 hold for some $l>0$, we investigate asymptotic stability with a necessary and sufficient condition by shifting our attention to the subset of functions in $S_{\psi}$ that tend to zero as $t \rightarrow \infty$, namely,

$$
S_{\psi}^{0}:=\left\{\phi \in S_{\psi} \mid \phi(t) \rightarrow 0 \text { as } t \rightarrow \infty\right\} .
$$

Since $S_{\psi}^{0}$ is a closed subset of $S_{\psi}$, the metric space $\left(S_{\psi}^{0}, d\right)$ is complete. Under the conditions of the next theorem, the zero solution of (1.1) is asymptotically stable.

Definition 2. The zero solution of (1.1) is asymptotically stable if it is stable at $t=0$ and a $\delta>0$ exists such that for any continuous functions $\psi:[m(0), 0] \rightarrow(-\delta, \delta)$, the solution $x$ with $x=\psi$ on $[m(0), 0]$ tends to zero as $t \rightarrow \infty$.

Recall here that B. Zhang was the first to establish necessary and sufficient condition for the stability of solutions of functional differential equations by the fixed point theory. The necessity of condition (2.8) below for the main stability result is due to him (see [25]).

Theorem 4. Suppose all of the conditions in Theorem 2 and Theorem 3 hold. Furthermore, suppose $g$ is continuously differentiable on $[-l, l]$ and 
$g^{\prime}(0) \neq 0$. Then the zero solution of $(1.1)$ is asymptotically stable if and only if

$$
\int_{0}^{t} H(v) d v \rightarrow \infty \text { as } t \rightarrow \infty
$$

Proof. First, suppose that (2.8) holds. We set

$$
K:=\sup _{t \geq 0}\left\{e^{-\int_{0}^{t} H(v) d v}\right\} .
$$

We show that $P: S_{\psi}^{0} \rightarrow S_{\psi}^{0}$ when the conditions of Theorems 2 and 3 hold, $l>0$ satisfies (2.11) and $\|\psi\|$ is sufficiently small. For $\delta>0$ satisfying (2.7), let $\psi:[m(0), 0] \rightarrow(-\delta, \delta)$ be a continuous function. Let $\phi \in S_{\psi}^{0}$. The proof of Theorem 3 shows that $\delta<l$ and $|(P \phi)(t)| \leq l$ for $t \in[m(0), \infty)$. Hence, $(P \phi)(t) \rightarrow 0$ would imply that $P$ maps $S_{\psi}^{0}$ into itself. To show that this is the case, we consider $|(P \phi)(t)|$. But first note for any $\phi \in S_{\psi}^{0}$ that

$$
|g(\phi(t))| \leq L|\phi(t)| \text { and }|\phi(t)-g(\phi(t))| \leq L|\phi(t)| \text {, }
$$

since $g(x)$ and $x-g(x)$ satisfy a Lipschitz condition on $[-l, l]$ with a common Lipschitz constant $L$ and $g(0)=0$. Because of this and (iii) of Theorem 3 , it follows from $(2.3)$ that

$$
\begin{aligned}
& |(P \phi)(t)| \\
& \leq\left\{|\psi(0)|+|G(0, \psi(-\tau(0)))|+\int_{-\tau(0)}^{0} H(s)|g(\psi(s))| d s\right\} e^{-\int_{0}^{t} H(v) d v} \\
& +|G(t, \phi(t-\tau(t)))|+\int_{t-\tau(t)}^{t} H(s)|g(\phi(s))| d s \\
& +\int_{0}^{t} e^{-\int_{s}^{t} H(v) d v} H(s)\left(\int_{s-\tau(s)}^{s} H(u)|g(\phi(u))| d u\right) d s \\
& +\int_{0}^{t} e^{-\int_{s}^{t} H(v) d v}\left\{\left|-a(s)+H(s-\tau(s))\left(1-\tau^{\prime}(s)\right)\right||g(\phi(s-\tau(s)))|\right. \\
& +H(s)|G(s, \phi(s-\tau(s)))|\} d s \\
& \quad+\int_{0}^{t} e^{-\int_{s}^{t} H(v) d v} H(s)|\phi(s)-g(\phi(s))| d s .
\end{aligned}
$$

Denote the six terms on the right hand side of $(2.10)$ by $I_{1}, I_{2}, \ldots, I_{6}$, respectively. It is obvious that the first term $I_{1}$ tends to zero as $t \rightarrow \infty$, by condition (2.8). Also, due to the conditions (1.2) and (1.3) and the facts 
that $\phi(t) \rightarrow 0$ and $t-\tau(t) \rightarrow \infty$ as $t \rightarrow \infty$, the second term $I_{2}$ in (2.10) tends to zero as $t \rightarrow \infty$. What is left to show is that each of the remaining terms in (2.10) tends to zero as $t \rightarrow \infty$.

Let $\phi \in S_{\psi}^{0}$ be fixed. For a given $\epsilon>0$, we choose $T_{0}>0$ large enough such that $t-\tau(t) \geq T_{0}$, implies $|\phi(s)|<\epsilon$ if $s \geq t-\tau(t)$. Therefore, the third term $I_{3}$ in (2.10) satisfies

$$
\begin{aligned}
& I_{3} \leq \int_{t-\tau(t)}^{t} H(s) L|\phi(s)| d s \\
& \leq L \epsilon \int_{t-\tau(t)}^{t} H(s) d s \leq L \alpha \epsilon .
\end{aligned}
$$

Thus, $I_{3} \rightarrow 0$ as $t \rightarrow \infty$. Now consider $I_{4}$. For the given $\epsilon>0$, there exists a $T_{1}>0$ such that $s \geq T_{1}$ implies $|\phi(s-\tau(s))|<\epsilon$. Thus, for $t \geq T_{1}$, the term $I_{4}$ in (2.10) satisfies

$$
\begin{aligned}
& I_{4} \leq \int_{o}^{T_{1}} e^{-\int_{s}^{t} H(v) d v} H(s)\left(\int_{s-\tau(s)}^{s} H(u) L|\phi(s)| d u\right) d s \\
& +\int_{T_{1}}^{t} e^{-\int_{s}^{t} H(v) d v} H(s)\left(\int_{s-\tau(s)}^{s} H(u) L|\phi(s)| d u\right) d s \\
& \leq \sup _{\sigma \geq m(0)}|\phi(\sigma)| L \int_{o}^{T_{1}} e^{-\int_{s}^{t} H(v) d v} H(s)\left(\int_{s-\tau(s)}^{s} H(u) d u\right) d s \\
& +L \epsilon \int_{T_{1}}^{t} e^{-\int_{s}^{t} H(v) d v} H(s)\left(\int_{s-\tau(s)}^{s} H(u) d u\right) d s .
\end{aligned}
$$

By (2.8), there exists $T_{2}>T_{1}$ such that $t \geq T_{2}$ implies

$$
\begin{aligned}
& \sup _{\sigma \geq m(0)}|\phi(\sigma)| L \int_{o}^{T_{1}} e^{-\int_{s}^{t} H(v) d v} H(s)\left(\int_{s-\tau(s)}^{s} H(u) d u\right) d s \\
= & \sup _{\sigma \geq m(0)}|\phi(\sigma)| L e^{-\int_{T_{2}}^{t} H(v) d v} \int_{o}^{T_{1}} e^{-\int_{s}^{T_{2}} H(v) d v} H(s)\left(\int_{s-\tau(s)}^{s} H(u) d u\right) d s \\
< & L \epsilon .
\end{aligned}
$$

Now, apply condition (iii) in Theorem 3 to have $I_{4}<L \epsilon+L \alpha \epsilon<2 L \epsilon$. Thus, $I_{4} \rightarrow 0$ as $t \rightarrow \infty$. Similarly, by using (1.2), (1.3) and condition (iii) in Theorem 3, then, if $t \geq T_{2}$ then the terms $I_{5}$ and $I_{6}$ in (2.10) satisfy

$$
\begin{aligned}
& I_{5} \leq \sup _{\sigma \geq m(0)}|\phi(\sigma)| e^{-\int_{T_{2}}^{t} H(v) d v} \int_{o}^{T_{1}} e^{-\int_{s}^{T_{2}} H(v) d v} \\
& \times\left\{L\left|-a(s)+H(s-\tau(s))\left(1-\tau^{\prime}(s)\right)\right|+E H(s)\right\} d s \\
& +\epsilon \int_{T_{1}}^{t} e^{-\int_{s}^{t} H(v) d v}\left\{L\left|-a(s)+H(s-\tau(s))\left(1-\tau^{\prime}(s)\right)\right|+E H(s)\right\} d s \\
& <L \epsilon+L \alpha \epsilon+\frac{\alpha g(l)}{l} \epsilon<\left(2 L+\frac{g(l)}{l}\right) \epsilon,
\end{aligned}
$$

and 


$$
\begin{aligned}
& I_{6} \leq \sup _{\sigma \geq m(0)}|\phi(\sigma)| L e^{-\int_{T_{2}}^{t} H(v) d v} \int_{o}^{T_{1}} e^{-\int_{s}^{T_{2}} H(v) d v} H(s) d s \\
& +L \epsilon \int_{T_{1}}^{t} e^{-\int_{s}^{t} H(v) d v} H(s) d s \\
& <L \epsilon+L \epsilon=2 L \epsilon .
\end{aligned}
$$

Thus, $I_{5}, I_{6} \rightarrow 0$ as $t \rightarrow \infty$. In conclusion $(P \phi)(t) \rightarrow 0$ as $t \rightarrow \infty$, as required. Hence $P$ maps $S_{\psi}^{0}$ into $S_{\psi}^{0}$.

Now we verify that $P$ is a contraction on $\left(S_{\psi}^{0}, d\right)$. In a similar way as in $[6$, Theorem 3.13], we let $\zeta \in[0, l]$ and define

$$
q(\zeta):=\min \left\{g^{\prime}(x):|x| \leq \zeta\right\} \text { and } Q(\zeta):=\max \left\{g^{\prime}(x):|x| \leq \zeta\right\} .
$$

As we shall see, the mapping $P$ defined by $(2.3)$ will be a contraction on $\left(S_{\psi}^{0}, d\right)$ provided

$$
\alpha Q(l)<q(l)
$$

where $\alpha$ is given by condition (iii) in Theorem 3. We may assume (2.11) holds for if it does not, we merely decrease the value of $l>0$ until it does. To see this, first notice from (iii) in Theorem 3 that $\alpha<1$. Thus,

$$
\alpha=1-\epsilon,
$$

for some $\epsilon \in(0,1)$. Clearly, $\lim _{\zeta \rightarrow 0} q(\zeta)=\lim _{\zeta \rightarrow 0} Q(\zeta)=g^{\prime}(0)$. Since $g$ is strictly increasing, $g^{\prime}(0) \neq 0$, and $g^{\prime}$ is continuous, a neighborhood of $x=0$ exists in which $g^{\prime}(x)>0$. Consequently, $Q(\zeta)>0$ for $0 \leq \zeta \leq l$. This and $\lim _{\zeta \rightarrow 0} Q(\zeta) \neq 0$ imply

$$
\lim _{\zeta \rightarrow 0} \frac{q(\zeta)}{Q(\zeta)}=\frac{\lim _{\zeta \rightarrow 0} q(\zeta)}{\lim _{\zeta \rightarrow 0} Q(\zeta)}=1 .
$$

Hence, there is a $\varsigma \in(0, l]$ such that

$$
\left|\frac{q(\zeta)}{Q(\zeta)}-1\right|<\epsilon
$$

for $0<\zeta<\varsigma$. Choosing a value for $\zeta$ from $(0, \varsigma)$, we have $(1-\epsilon) Q(\zeta)<$ $q(\zeta)$. This, along with (2.12), yields

$$
\alpha Q(\zeta)=(1-\epsilon) Q(\zeta)<q(\zeta)
$$


Replacing the original value of $l$ with $l=\zeta$, we obtain (2.11). Note that the conditions in Theorems 2 and 3 will still hold with this smaller $l$.

In the ensuing argument, bounds on the derivatives of $g(x)$ and $b(x):=$ $x-g(x)$ on the interval $[-l, l]$ are used. By $(i)$ in Theorem 3 and the definition of $Q, 0 \leq g^{\prime}(x) \leq Q(l)$. By $(i)$ and $(i i)$ in Theorem $3, b$ is nondecreasing on $[-l, l]$. This and $g^{\prime}(x) \geq q(l)$ imply $0 \leq b^{\prime}(x) \leq 1-q(l)$. Let $\phi, \eta \in S_{\psi}^{0}$. By (2.3) and the Mean Value Theorem, we have

$$
\begin{aligned}
& |(P \phi)(t)-(P \eta)(t)| \\
& \leq E|\phi(t-\tau(t))-\eta(t-\tau(t))| \\
& +\int_{t-\tau(t)}^{t} H(s)|g(\phi(s))-g(\eta(s))| d s \\
& +\int_{0}^{t} e^{-\int_{s}^{t} H(v) d v} H(s) \int_{s-\tau(s)}^{s} H(u)|g(\phi(u))-g(\eta(u))| d u d s \\
& +\int_{0}^{t} e^{-\int_{s}^{t} H(v) d v}\left\{\left|-a(s)+H(s-\tau(s))\left(1-\tau^{\prime}(s)\right)\right|\right. \\
& \times|g(\phi(s-\tau(s)))-g(\eta(s-\tau(s)))|+E H(s)|\phi(s-\tau(s))-\eta(s-\tau(s))|\} d s \\
& \left.+\int_{0}^{t} e^{-\int_{s}^{t} H(v) d v} H(s)|b(\phi(s))-b(\eta(s))|\right\} d s \\
& \leq[\alpha Q(l)+(1-q(l))]\|\phi-\eta\|=\rho\|\phi-\eta\|,
\end{aligned}
$$

for all $t>0$, where $\rho=\alpha Q(l)+(1-q(l))$. This implies $\|P \phi-P \eta\| \leq$ $\rho\|\phi-\eta\|$. Note $\rho \in(0,1)$. Consequently, for a continuous $\psi:[m(0), 0] \rightarrow$ $(-\delta, \delta), P$ has a unique fixed point $x \in S_{\psi}^{0}$. By Lemma $1, x$ is the unique continuous solution of (1.1) with $x(t)=\psi(t)$ on $[m(0), 0]$. By virtue of $x \in S_{\psi}^{0}, x$ tends to 0 as $t \rightarrow \infty$. By Theorem 3 , the zero solution is stable at $t=0$. This shows that the zero solution of (1.1) is asymptotically stable if $(2.8)$ holds.

Conversely, suppose (2.8) fails. Then, there exists a sequence $\left\{t_{n}\right\}$, $t_{n} \rightarrow \infty$ as $n \rightarrow \infty$ such that $n \rightarrow \infty \lim \int_{0}^{t_{n}} H(v) d v=\beta$ for some $\beta \in \mathbf{R}^{+}$. We may also choose a positive constant $J$ satisfying

$$
-J \leq \int_{0}^{t_{n}} H(v) d v \leq J
$$

for all $n \geq 1$. To simplify our expressions, we define

$$
\theta(s):=(l E+L) H(s)+g(l)\left\{\left|-a(s)+H(s-\tau(s))\left(1-\tau^{\prime}(s)\right)\right|\right.
$$




$$
\left.+H(s) \int_{s-\tau(s)}^{s} H(u) d u\right\}
$$

for all $s \geq 0$. By by condition (iii) in Theorem 3, we have

$$
\int_{0}^{t_{n}} e^{-\int_{s}^{t_{n}} H(v) d v} \theta(s) d s \leq \alpha g(l)+L
$$

This yields

$$
\int_{0}^{t_{n}} e^{\int_{0}^{s} H(v) d v} \theta(s) d s \leq(\alpha g(l)+L) e^{\int_{0}^{t_{n}} H(v) d v} \leq(g(l)+L) e^{J} .
$$

The sequence $\left\{\int_{0}^{t_{n}} e^{\int_{0}^{s} H(v) d v} \theta(s) d s\right\}$ is bounded, so there exists a convergent subsequence. For brevity of notation, we may assume that

$$
\lim _{n \rightarrow \infty} \int_{0}^{t_{n}} e^{\int_{0}^{s} H(v) d v} \theta(s) d s=\gamma,
$$

for some $\gamma \in \mathbf{R}^{+}$and choose a positive integer $m$ so large that

$$
\int_{t_{m}}^{t_{n}} e^{\int_{0}^{s} H(v) d v} \theta(s) d s<\delta_{0} / 4 K
$$

for all $n \geq m$, where $\delta_{0}>0$ satisfies

$$
\left\{\delta_{0}(1+E)+g\left(\delta_{0}\right) \int_{t_{m}-\tau\left(t_{m}\right)}^{t_{m}} H(s) d s\right\} K e^{J} \leq(1-\alpha) g(l) .
$$

If we replace $l$ by 1 in the proof of Theorem 3 , we have $|x(t)| \leq 1$ for $t \geq t_{m}$. Now we consider the solution $x(t)=x\left(t, t_{m}, \psi\right)$ of (1.1) with $\psi\left(t_{m}\right)=\delta_{0}$ and $|\psi(s)| \leq \delta_{0}$ for $s \leq t_{m}$. We may choose $\psi$ so that

$$
\psi\left(t_{m}\right)-G\left(t_{m}, \psi\left(t_{m}-\tau\left(t_{m}\right)\right)\right)-\int_{t_{m}-\tau\left(t_{m}\right)}^{t_{m}} H(s) g(\psi(s)) d s \geq \frac{1}{2} \delta_{0} .
$$

It follows from $(2.3)$ with $x(t)=(P x)(t)$ that for $n \geq m$

$$
\begin{aligned}
& \left|x\left(t_{n}\right)-G\left(t_{n}, x\left(t_{n}-\tau\left(t_{n}\right)\right)\right)-\int_{t_{n}-\tau\left(t_{n}\right)}^{t_{n}} H(s) g(x(s)) d s\right| \\
& \geq \frac{1}{2} \delta_{0} e^{-\int_{t_{m}}^{t_{n}} H(v) d v}-\int_{t_{m}}^{t_{n}} e^{-\int_{s}^{t_{n}} H(v) d v} \theta(s) d s \\
& =\frac{1}{2} \delta_{0} e^{-\int_{t_{m}}^{t_{n}} H(v) d v}-e^{-\int_{0}^{t_{n}} H(v) d v} \int_{t_{m}}^{t_{n}} e^{\int_{0}^{s} H(v) d v} \theta(s) d s \\
& =e^{-\int_{t_{m}}^{t_{n}} H(v) d v}\left(\frac{1}{2} \delta_{0}-e^{-\int_{0}^{t_{m}} H(v) d v} \int_{t_{m}}^{t_{n}} e^{\int_{0}^{s} H(v) d v} \theta(s) d s\right)
\end{aligned}
$$




$$
\geq e^{-\int_{t_{m}}^{t_{n}} H(v) d v}\left(\frac{1}{2} \delta_{0}-K \int_{t_{m}}^{t_{n}} e^{\int_{0}^{s} H(v) d v} \theta(s) d s\right)
$$

$$
\geq \frac{1}{4} \delta_{0} e^{-\int_{t_{m}}^{t_{n}} H(v) d v} \geq \frac{1}{4} \delta_{0} e^{-2 J}>0 .
$$

On the other hand, if the zero solution of (1.1) is asymptotically stable, then $x(t)=x\left(t, t_{m}, \psi\right) \rightarrow 0$ as $t \rightarrow \infty$. Since $t_{n}-\tau\left(t_{n}\right) \rightarrow \infty$ as $n \rightarrow \infty$ and condition (iii) in Theorem 3 holds, we have

$$
x\left(t_{n}\right)-G\left(t_{n}, x\left(t_{n}-\tau\left(t_{n}\right)\right)\right)-\int_{t_{n}-\tau\left(t_{n}\right)}^{t_{n}} H(s) g(x(s)) d s \rightarrow 0 \text { as } n \rightarrow \infty,
$$

which contradicts (2.13). Hence condition (2.8) is necessary for the asymptotic stability of the zero solution of (1.1). The proof is complete.

Remark 1. Obviously, if $G(t, x)=0$, Theorem 4 extends Theorem 1.

\section{An Example}

In this section, we give an example to illustrate the application of Theorem 4 .

Example 1. Consider the following totally nonlinear neutral differential equation with variable delay

$$
\frac{d}{d t} x(t)=-a(t) g(x(t-\tau(t)))+\frac{d}{d t} G(t, x(t-\tau(t))),
$$

where $\tau(t)=0.172 t, a(t)=0.391 /(0.828 t+1), g(x)=\sin x, G(t, x)=$ $0.096 \sin (x / 3)$. Then the zero solution of (3.1) is asymptotically stable.

Proof. Choosing $l=\pi / 3, k=6$ and $H(t)=1 /(t+1)$, clearly, condition (2.8) holds. Furthermore, we have $L=2, E=0.032, g(0)=0$, $g(l)=\sqrt{3} / 2, g^{\prime}(0)=1$ and $G(t, 0)=0$. Obviously, $g$ is odd and strictly increasing on $[-\pi / 3, \pi / 3], x-g(x)$ is non-decreasing on $[0, \pi / 3]$,

$$
\begin{aligned}
& \int_{t-\tau(t)}^{t} H(s) d s=\int_{0.828 t}^{t} \frac{1}{s+1} d s=\ln \left(\frac{t+1}{0.828 t+1}\right)<0.189, \\
& \int_{0}^{t} e^{-\int_{s}^{t} H(v) d v} H(s)\left(\int_{s-\tau(s)}^{s} H(u) d u\right) d s<0.189
\end{aligned}
$$

and 


$$
\begin{aligned}
& \int_{0}^{t} e^{-\int_{s}^{t} H(v) d v}\left|-a(s)+H(s-\tau(s))\left(1-\tau^{\prime}(s)\right)\right| d s \\
& =\int_{0}^{t} e^{-\int_{s}^{t} \frac{1}{v+1} d v}\left|-\frac{0.391}{0.828 s+1}+\frac{0.828}{0.828 s+1}\right| d s \\
& <\frac{0.437}{0.828} \int_{0}^{t} e^{-\int_{s}^{t} \frac{1}{v+1} d v} \frac{1}{s+1} d s<0.528 .
\end{aligned}
$$

It is easy to see that all the conditions of Theorems 2, 3 and 4 hold for $\alpha=[(\pi / 3) /(\sqrt{3} / 2)](0.064)+0.189+0.189+0.528 \simeq 0.984<1$. Thus, Theorem 4 implies that the zero solution of (3.1) is asymptotically stable.

Acknowledgment. The authors would like to thank the anonymous referee for his/her valuable comments.

\section{References}

[1] A. Ardjouni, A. Djoudi, Fixed points and stability in linear neutral differential equations with variable delays. Nonlinear Analysis 74, pp. 2062-2070, (2011).

[2] A. Ardjouni, A. Djoudi, Stability in nonlinear neutral differential with variable delays using fixed point theory, Electronic Journal of Qualitative Theory of Differential Equations, No. 43, pp. 1-11, (2011).

[3] A. Ardjouni, A. Djoudi, Fixed point and stability in neutral nonlinear differential equations with variable delays, Opuscula Mathematica, Vol. 32, No. 1, pp. 5-19, (2012).

[4] A. Ardjouni, A. Djoudi, I. Soualhia, Stability for linear neutral integrodifferential equations with variable delays, Electronic journal of Differential Equations, No. 172, pp. 1-14, (2012).

[5] A. Ardjouni, A. Djoudi, Fixed points and stability in nonlinear neutral Volterra integro-differential equations with variable delays, Electronic Journal of Qualitative Theory of Differential Equations, No. 28, pp. $1-13,(2013)$

[6] L. C. Becker, T. A. Burton, Stability, fixed points and inverse of delays, Proc. Roy. Soc. Edinburgh 136A, pp. 245-275, (2006). 
[7] T. A. Burton, Fixed points and stability of a nonconvolution equation, Proceedings of the American Mathematical Society 132, pp. 36793687, (2004).

[8] T. A. Burton, Stability by Fixed Point Theory for Functional Differential Equations, Dover Publications, New York, (2006).

[9] T. A. Burton, Liapunov functionals, fixed points, and stability by Krasnoselskii's theorem, Nonlinear Studies 9, pp. 181-190, (2001).

[10] T. A. Burton, Stability by fixed point theory or Liapunov's theory: A comparison, Fixed Point Theory 4, pp. 15-32, (2003).

[11] T. A. Burton, T. Furumochi, A note on stability by Schauder's theorem, Funkcialaj Ekvacioj 44, pp. 73-82, (2001).

[12] T. A. Burton, T. Furumochi, Fixed points and problems in stability theory, Dynamical Systems and Applications 10, pp. 89-116, (2001).

[13] T. A. Burton, T. Furumochi, Asymptotic behavior of solutions of functional differential equations by fixed point theorems, Dynamic Systems and Applications 11, pp. 499-519, (2002).

[14] T. A. Burton, T. Furumochi, Krasnoselskii's fixed point theorem and stability, Nonlinear Analysis 49, pp. 445-454, (2002).

[15] Y. M. Dib, M. R. Maroun, Y. N. Raffoul, Periodicity and stability in neutral nonlinear differential equations with functional delay, Electronic Journal of Differential Equations, Vol. No. 142, pp. 1-11, (2005).

[16] A. Djoudi, R. Khemis, Fixed point techniques and stability for neutral nonlinear differential equations with unbounded delays, Georgian Mathematical Journal, Vol. 13, No. 1, pp. 25-34, (2006).

[17] C. H. Jin, J. W. Luo, Stability of an integro-differential equation, Computers and Mathematics with Applications 57, pp. 1080-1088, (2009).

[18] C. H. Jin, J. W. Luo, Stability in functional differential equations established using fixed point theory, Nonlinear Anal. 68, pp. 3307$3315,(2008)$.

[19] C. H. Jin, J. W. Luo, Fixed points and stability in neutral differential equations with variable delays, Proceedings of the American Mathematical Society, Vol. 136, Nu. 3, pp. 909-918, (2008). 
[20] J. Luo, Fixed points and exponential stability for stochastic VolterraLevin equations, Journal of Computational and Applied Mathematics, Vol. 234, Issue 3, 1 June 2010, pp. 934-940, (2010).

[21] Y. N. Raffoul, Stability in neutral nonlinear differential equations with functional delays using fixed-point theory, Math. Comput. Modelling 40, pp. 691-700, (2004).

[22] D. R. Smart, Fixed point theorems, Cambridge Tracts in Mathematics, No. 66. Cambridge University Press, London-New York, (1974).

[23] J. A. Yorke, Asymptotic stability for one dimensional differential delay equations, J. Diff. Eqns 7, pp. 189-202, (1970).

[24] B. Zhang, Fixed points and stability in differential equations with variable delays, Nonlinear Anal. 63, pp. e233-e242, (2005).

[25] B. Zhang, Contraction mapping and stability in a delay differential equation, Dynamical systems and appl. 4, pp. 183-190, (2004).

\author{
Abdelouaheb Ardjouni \\ Faculty of Sciences and Technology, \\ Department of Mathematics and Informatics, \\ University Souk Ahras, \\ P. O. Box 1553, \\ Souk Ahras, 41000, \\ Algeria \\ e-mail : abd_ardjouni@yahoo.fr \\ and
}

\author{
Ahcene Djoudi \\ Applied Mathematics Lab, \\ Faculty of Sciences, \\ Department of Mathematics, \\ University Annaba, \\ P. O. Box 12, Annaba 23000, \\ Algeria \\ e-mail : adjoudi@yahoo.com
}

